\title{
Christoph Haffter
}

\section{Szenen der Selbstenttäuschung}

\author{
Hanns Eislers Die rote und die weiße Rose \\ nach Li Bai und die Antinomien \\ der Kriegslyrik
}

\section{Scenes of Self-Deception. Hanns Eisler's The Red and White Rose after Li Bai, and the Antinomies of War Poetry}

\begin{abstract}
In 1917, the young Hanns Eisler composed a song to a Li Bai text as part of an anti-war oratorio that he never completed. How could one transform the horrors of war into art without transfiguring them, and without rendering senseless slaughter meaningful? This must have been the question that Eisler was not able to answer properly. He based his work on the translation by Klabund, whose poetry contradicts Eisler's pacifist intentions. An analysis of the song shows how the central idea of Li Bai's poem - a scene of self-deception - resonates with the techniques of musical self-criticism that arise from this contradiction, and that would become one of the key characteristics of Eisler's later style. The non-affirmative, doubting character of his music is interpreted as a response to three interrelated philosophical antinomies: the antinomy of war poetry, of pacifism and of revolutionary art. It is argued here that Eisler's most convincing works express these antinomies as a struggle between self-deception and disillusion, between hope and disenchantment. The truth of Eisler's music lies in the elaboration of this antagonism that is already present in his earliest encounter with the poetry of Li Bai.
\end{abstract}

Der Komponist Hanns Eisler (1898-1962) war vom Kriege nie begeistert. Die Verurteilung des Kriegs ist ein Motiv, um das etliche seiner Werke kreisen. Das hat sicherlich auch biografische Gründe: Gegen seine Überzeugung wurde Eisler als Jugendlicher in die österreichisch-ungarische Armee eingezogen, in die Schützengräben der italienischen Front geschickt, überstand Giftgasangriffe und lag monatelang verletzt im Lazarett. ${ }^{1}$ In dieser Umgebung soll er 1917 die Arbeit an einer seiner ersten Kompositionen aufgenommen haben: einem Oratorium gegen den Krieg unter dem Titel Dumpfe

DOI: $10.26045 /$ po-015

1 Vgl. Thomas Phleps:»Der müde Soldat«. Hanns Eisler und der Erste Weltkrieg, in: Musik bezieht Stellung. Funktionalisierungen der Musik im Ersten Weltkrieg, hg. von Stefan Hanheide, Dietrich Helms, Claudia Glunz und Thomas F. Schneider, Göttingen: Universitätsverlag Osnabrück, 2013, S. 403-428. 
Trommel und berauschtes Gong. ${ }^{2}$ Der Titel ist einer Sammlung deutscher Nachdichtungen chinesischer Kriegslyrik entlehnt, die der Dichter Klabund (1890-1928) verfasst hat. Eisler hat das Oratorium nicht vollendet, zwei Klavierlieder sind jedoch erhalten, vermutlich als Vorarbeiten zu Liedern mit Ensemble- oder gar Orchesterbegleitung. Eines dieser Lieder verarbeitet die Nachdichtung eines Gedichts von Li Bai:

Die weiße und die rote Rose

Während ich mich über meine Stickerei am Fenster bückte, Stach mich meine Nadel in den Daumen. Weiße Rose,

Die ich stickte,

Wurde rote Rose.

In der kriegerischen Weite bei des Vaterlandes Söhnen

Weilt mein Freund, vergießt vielleicht sein Blut.

Rossehufe hör ich dröhnen.

Ists sein Pferd? Es ist mein Herz, das wie ein Fohlen tut.

Tränen fallen mir aus meinen Blicken

Übern Rahmen in die Stickerein.

Und ich will die Tränen in die Seide sticken,

Und sie sollen weiße Perlen sein. ${ }^{3}$

\section{Die Selbsttäuschung: eine Analyse}

Hanns Eisler soll Theodor W. Adorno (1903-1969) einmal erklärt haben, dass man Lieder stets von der Pointe her komponieren müsse. ${ }^{4}$ Ein solches Vorgehen kann man schon in diesem Jugendwerk erkennen: Es handelt sich um eine Szene der Selbsttäuschung. Eisler hätte für sein Anti-Kriegsoratorium auf grausige Verse aus Klabunds Sammlung zurückgreifen können, die ungeschönt die Versehrungen des Kriegs beschreiben: Wie Schakale sich um das Hirn toter Soldaten beißen, wie in der Winterschlacht die im Stiefel erfrorenen Gliedmaßen abgeschnitten werden, wie der Getöteten Eingeweide von den Bäumen hängen. Doch Eisler vertont ein Gedicht, das um eine

2 Zur Quellenlage und zum unvollendeten Oratorium vgl. Christian Martin Schmidt: Einleitung, in: Hanns Eisler: Lieder für Singstimme und Klavier 1917-1921, hg. von Julia Rittig-Becker und Christian M. Schmidt, Wiesbaden: Breitkopf \& Härtel, 2009 (Gesamtausgabe. Serie 3, Bd. 1), S. XI-XVI. Vgl. auch Phleps: »Der müde Soldat«, S. 407.

3 Zitiert nach der Transkription der Originalausgabe unter http://klabund.eu/wp/li-tai-pe-nach dichtungen-von-klabund/ (letzter Zugriff auf alle Links in diesem Beitrag am 06.08.2020).

Theodor W. Adorno: Notizen über Eisler [1992], in: Hanns Eisler, hg. von Albrecht Dümling, Frankfurt am Main: Stroemfeld, 2010 (Querstand. Musikalische Konzepte, Bd. 5/6), S. 73-83, hier S. 78. 
intime Enttäuschungsszene herum konstruiert ist: Die daheimgebliebene Frau, das lyrische Ich, glaubt das Pferdegetrappel zu hören, das ihr die Rückkehr ihres Mannes aus dem fernen Krieg verspricht; doch sie durchschaut ihr eigenes Wunschdenken und erkennt, dass sie, sich selbst täuschend, das heftige Schlagen ihres Herzens für das Geräusch der Hufe nahm. Die Struktur der Selbsttäuschung zeigt sich darin, dass Ursache und Wirkung des Vorgangs ineinander übergehen: Das Gedicht setzt mit dem versehentlichen Stich ein, den sich das lyrische Ich bei der Stickarbeit zufügt. Das Blut, das sogleich von ihrem Daumen auf die gestickte weiße Rose tropft, mahnt sie an ihren Geliebten, der womöglich im Kampf verblutet. Die Unachtsamkeit, welche die Frau zu solcher Selbstverletzung verleitet, ist aber wohl bereits darauf zurückzuführen, dass die Stickerin nicht recht bei der Sache war, weil sie, am Fenster wie an einer Schwelle zur Außenwelt sitzend, an ihren Geliebten in der Ferne dachte. Die sich rot einfärbende Rose, die dem lyrischen Ich die Gefahr bedeutet, in welcher der Geliebte schwebt, lässt ihr Herz vor Kummer höher schlagen; das Schlagen ihres Herzens vergegenwärtigt ihr wiederum den Geliebten zu Pferd, was nun abermals ihr Herz, nun aber vor Freude, heftiger pochen lässt. So ziehen sich die Wirkungsschlaufen der Sehnsucht zum Knoten zusammen, bis er platzt: Die Rückkopplungen brechen ab, die Selbsttäuschung verfliegt und das lyrische Ich fällt aus der Illusion in die Welt der Tatsachen zurück, ganz so wie ihre Tränen in den Stoff ihrer Stickarbeit fallen, um sich dort gleich wieder in Illusion zu wandeln und vom Schein einer besseren Welt zu zeugen.

Eisler richtet sein Lied, gleich einer kurzen Szene, an dieser Pointe aus. Das Lied verarbeitet - wie das Stickwerk im Gedicht - die Wirkung eines lautmalerischen Trugs: des Getrappels, das sich aus dem musikalischen Gedanken an den Geliebten in den kriegerischen Weiten (T. 15-21) entwickelt, um sich von Takt 22 bis zur Fermate in Takt 28 heftig zu steigern. Diese Phase, in der sich das lyrische Ich in den Schein hineinsteigert, bildet den Mittelteil des Lieds, der, harmonisch um cis-Moll zentriert, sich abgrenzt und umfasst wird vom einem ersten in b-Moll notierten Abschnitt und einem Schlussteil, der auf f-Moll endet - wobei die harmonischen Verhältnisse oft vieldeutig sind, darin den letzten tonalen Liedern Schönbergs, etwa den 8 Liedern op. 6 verwandt, deren drittes Lied mit dem Titel »Verlassen« Eisler auch im Charakter als Vorbild hätte dienen können.

Eisler folgt somit der Dreiteiligkeit des Gedichts, die sich aus dem Kontrast ergibt zwischen der drängenden Zeit der sich verselbständigenden Scheinwelt im Mittelteil und der richtungslosen Zeit des Wartens in der scheinlosen Wirklichkeit, der Stickerei zu Beginn und am Ende. Der Schlussabschnitt nimmt jedoch Momente der Illusion in sich auf, er verarbeitet sozusagen die Tränen, welche die erhitzte Einbildungskraft hervortrieb, ins Gewebe der profanen Wirklichkeit fortdauernder Arbeit. Diese wird repräsentiert durch das melancholische Grundmotiv, mit dem das Lied in der rechten Hand des Klaviers einsetzt. Es hat in seinem Auf- und Absteigen zyklischen Charakter und kann als chromatische Umspielung eines verminderten Septakkords (es/ges/a/c) gehört werden, der auf ein abwesendes b-Moll bezogen ist, ohne jedoch auf eine solche Auflösung zu drängen. Dieses Motiv des wartenden Stickens, mit dem auch der Gesang anhebt und endet, durchzieht fast unverändert das gesamte Lied, wobei es oftmals in begleitende Texturen eingeht. Einzig während der Geräuschhalluzination fehlt es gänzlich, das lyrische Ich legt sozusagen die Arbeit in den Schoß. 
II Die rote und die weiße Rose

(Klabund nach Li-Tai-Pe)
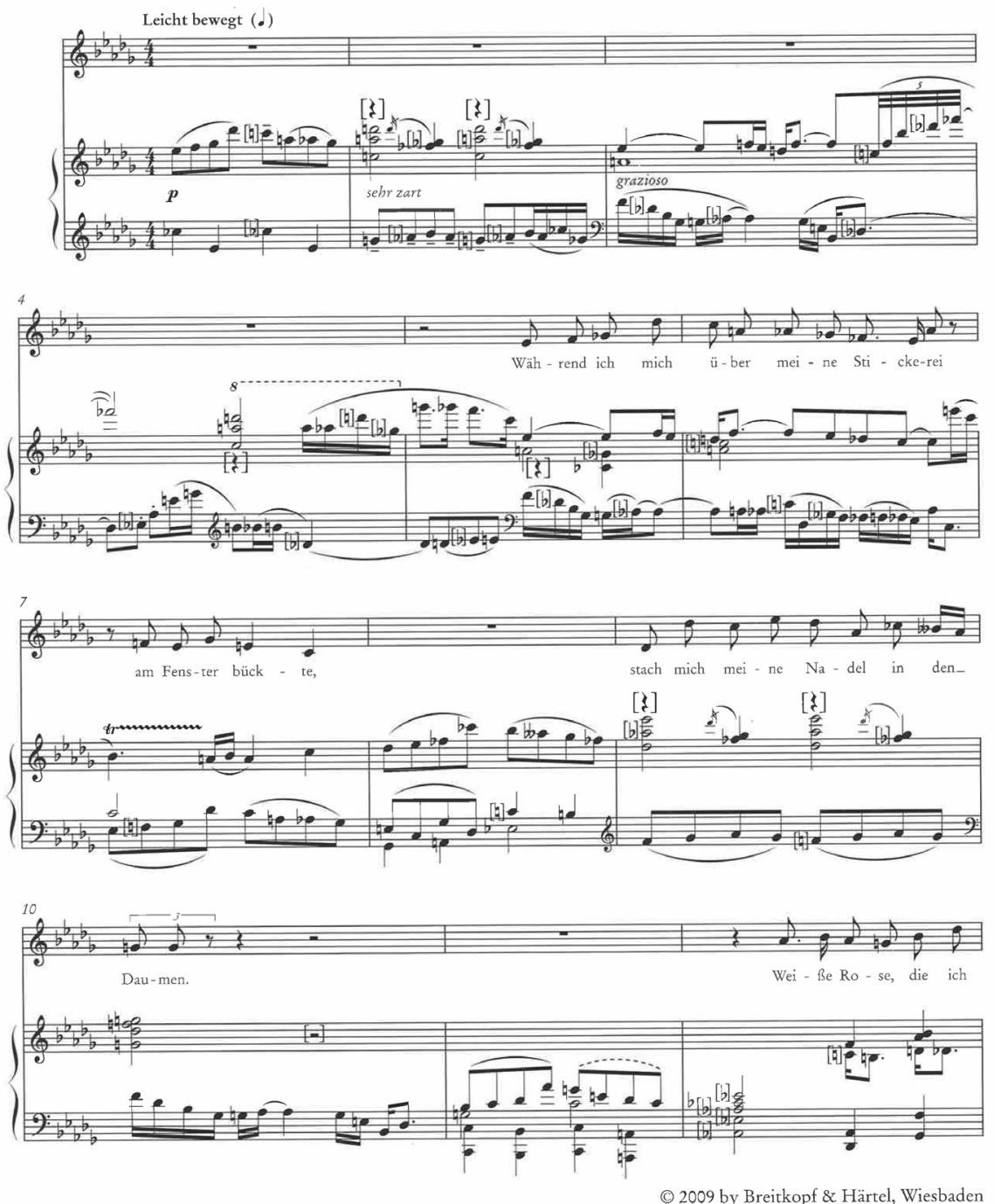

Abb. 1: Hanns Eisler: Die rote und die weiße Rose, T. 1-12 (๔ 2009 Breitkopf \& Härtel, Wiesbaden)

Bereits im zweiten Takt erscheint, harmonisch unvermittelt, als zweites Motiv ein wiederholter Akkordwechsel, der sehr zart zu spielen ist: ein gläserner Akkord, in welchem zwei Quarten im Abstand einer großen Sexte, später im Lied (T. 9, T. 37 f.) zwei Quinten übereinander gestapelt werden, um auf eine große Sekunde mit fallendem Quintvorschlag zu wechseln, während in der linken Begleithand die kreisende Achtelbewegung fortgesetzt wird. Auf das Ende des Lieds vorgreifend, lässt sich der durchsichtig-hohle Klangcharakter dieses Materials auf das Motiv der in die Stickerei einge- 
arbeiteten Tränenperlen beziehen; es ist exterritoriales Material, das sich nicht zerlegen und entwickeln lässt und auch keine Begleitfunktion übernehmen kann, sondern so unbeweglich wie folgenlos das Geschehen unterbricht. Im dritten Takt erscheint in der linken Hand ein weiteres Grundmotiv des Lieds, das unter einem Tritonus in der rechten Hand eine Sechzehntelbewegung in fallenden Terzen umfasst, die kurz von einer charakteristischen Schwelle, einer chromatischen Gegenbewegung, aufgefangen wird, um sogleich weiter zu fallen. Dem Motiv ist eine gewisse Komik eigen, etwas von dem kindlichen Unernst, der im Wort >kullern « mitschwingt - man mag an die chromatisch aufgefangenen Terzfälle des Ragtime denken, etwa an Scott Joplins Cascades oder an Debussys Children's Corner. Dieser unernste Charakter ist zu Beginn noch nicht vorherrschend, spätestens aber wenn dieses Motiv im Anschluss an den Vers »stach ich meine Nadel in den Daumen« alleine im Bass erklingt, tritt seine komische Seite deutlich hervor. Das Motiv steht für einen Zug ins Groteske, der das Lied in die Nähe der späteren Morgenstern-Vertonungen Eislers bringt. ${ }^{5}$

Während die melancholische Zerstreutheit des lyrischen Ichs in der dichten und harmonisch gewagten Polyphonie im Klavier der ersten Takte einen durchaus ernsten Widerschein findet, wird die Kadenz im siebten Takt auffallend artig von Triller und Vorschlag eingeleitet; eine Übertreibung jenes Preziösen oder Manierlichen, von dem Klabunds Nachdichtungen nie ganz frei sind: Es klingt, als schämte sich die Musik für einen Moment des Klischees fernöstlicher Damenhaftigkeit, das das Gedicht mit der häuslichen Stickarbeit evoziert.

Die auf das Ungeschick des Nadelstichs folgende Verfärbung der Rose nimmt Klabund zum Anlass, die deutsche Sprache sachte zu verfremden, indem er die Artikel weglässt und Zeilenumbrüche setzt:

Weiße Rose,

die ich stickte,

wurde rote Rose.

Die Sprache erhält so etwas von jener rätselhaften Schlichtheit, die Klabund womöglich mit der chinesischen Kunstsprache assoziiert haben mag; stellt man sie sich doch als eine Aneinanderreihung von Zeichen vor, die weder konjugiert noch von Artikeln begleitet werden und deren syntaktisch-semantische Bezüge daher in einer gewissen Schwebe gehalten sind - wobei gesagt werden muss, dass weder Klabund noch ich selbst die chinesische Sprache beherrschen. Klabunds Verfremdung wird im Vergleich mit Bethges Übertragung dieser Stelle deutlich:

Da stach ich mich, - und rotes Blut rann auf

Die weisse, weisse Rose, die ich stickte,

Und eine rote Rose ward daraus. ${ }^{6}$

6 Hans Bethge: Die chinesische Flöte, Leipzig: Insel-Verlag, 1907, S. 30. 
Während Bethges Verse mit der emphatischen Wortwiederholung und dem altertümlichen >ward ‘ zur Behäbigkeit tendieren, riskiert Klabunds Kunstgriff, dass der Vers das Damenhafte ins Dämliche kippen lässt: Wer so spricht, ist nicht tief, sondern einfältig. Eisler nimmt das Spärliche der Dichtung auf, setzt vor und nach dem Satz (T. 11-14) das identische Stickereimotiv, das nun nach as-Moll geführt wird; ein einzelner Ton jedoch, das $g$ in Takt 11, ist in der Wiederholung tiefalteriert, sodass die Stimme die nunmehr >rote Rose im verdunkelten Intervall ges-c singt.

Der Mittelteil, der daran ansetzt, öffnet den dichterischen Raum aufs Imaginäre. In diesem Sinne lässt sich auch die enharmonische Umdeutung von as zu gis in Takt 15 deuten, sodass nun gewissermaßen derselbe Klang anders aufgefasst, imaginär überhöht wird. Zusammen mit dem drängenden Motiv der rechten Hand, das sich ins Hufgetrappel verwandeln wird, entwickelt das Stickereimotiv in der Begleitung nun harmonische Spannung und treibt in Richtung cis-Moll; montageartig tritt der Gedanke ans Blutvergießen in den kriegerischen Weiten ein, ein Staccato-Marsch, der leise, aber marcato gesetzt ist - das für den späteren Eisler typische Spitzige der Musik, ein Ineinander von Schrecken und Naivität, ist hier bereits fühlbar. Das punktierte Motiv der Hufe wird bis zur Fermate gesteigert, auch der Gesang erhitzt sich, was nicht recht überzeugen will, vielleicht auch weil Eisler einen Widersinn komponiert, wenn er die Stimme statt der fragenden Betonung »Ist's sein Pferd? « ein grotesk aufgeregtes »Ist's sein Pferd? « singen lässt, sodass man glauben könnte, das Pferd nahe ohne Reiter. Sodann verfliegt der Trug nach der Fermate, die musikalische Spannung fällt in sich zusammen, ungut erklingt im Klavier ein tiefes cis mit chromatischem Schleifer, über dem der Gesang, einer Sprechstimme angeglichen, ernüchtert einsehen muss: »Es ist mein Herz, das wie ein Fohlen tut «.

Die Reprise ist in f-Moll notiert, die Musik hält jedoch an der enharmonischen Umdeutung fest; der Gesang bringt das Stickereimotiv so, wie es zu Beginn des Mittelteils im Klavier erklingt, während das dort drängende Motiv der Stimme nun in
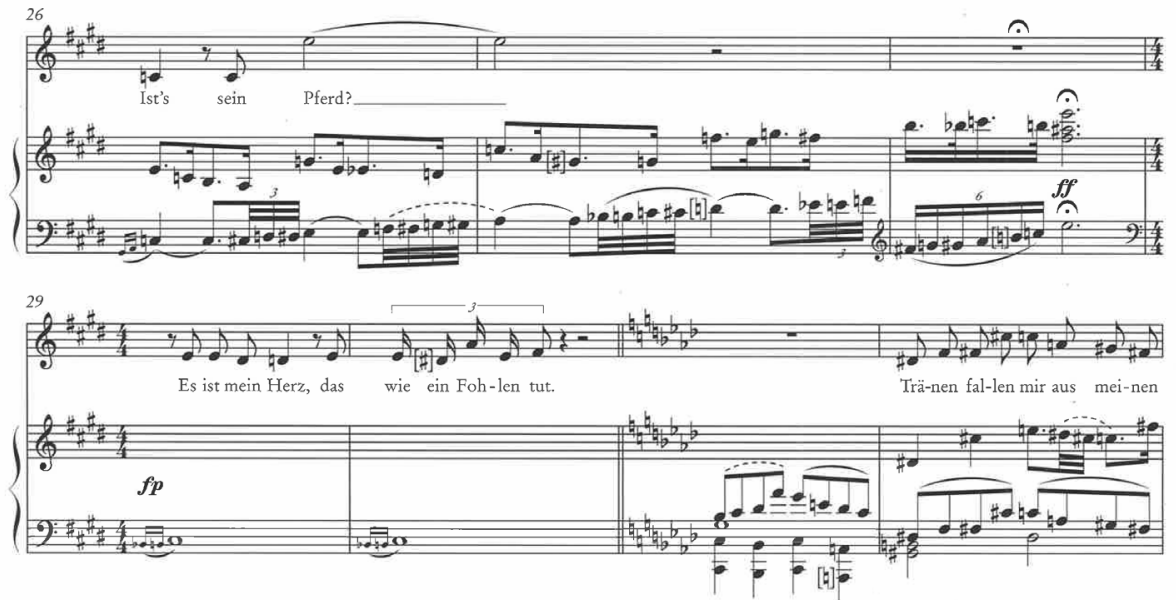

Abb. 2: Hanns Eisler: Die rote und die weiße Rose, T. 26-32 (๔ 2009 Breitkopf \& Härtel, Wiesbaden) 
die Klavierbegleitung eingeht und nur mehr als Nachklang erscheint. Das musikalische Material der Tränenperlen, das sieben Takte vor Schluss wieder erklingt, die gläsernen Akkordwechsel, sind der Anlass zur enharmonischen Rückdeutung; die Musik kühlt ab und scheint mit der Rückkehr des kullernden Motivs in Takt 39 gar wieder von sich selbst Abstand zu nehmen. Mit dem Wunsch "Und es sollen weiße Perlen sein ... « wird das Hauptmotiv ein letztes Mal bemüht und in einen nicht ganz von sich überzeugten Schluss mit arpeggierenden f-Moll-Akkorden geführt: Die Schlussgeste ist aufgesetzt.

\title{
Antinomien der Kriegslyrik
}

Die Analyse des Lieds lässt eine gewisse Unentschlossenheit hervortreten, mit der Eisler den Text Klabunds behandelt: Er scheint ihn, und damit seine eigene Musik, nicht ganz ernst nehmen zu können. Ich möchte im Folgenden einige Überlegungen anstellen, wie sich dieser Charakter der Selbstdistanz deuten lässt, der Eislers Vertonung der Dichtung Li Bais auszeichnet.

Wahrscheinlich hat ein gewisses Unbehagen mit der literarischen Vorlage Eisler zum Abbruch des Kriegsoratoriums gedrängt. Ein deutliches Zeichen dafür ist die Kürzung, die Eisler am Text vornimmt: Klabunds »bei des Vaterlandes Söhnen« in der ersten Strophe kommt in Eislers Lied verständlicherweise nicht vor. Das Detail weist auf eine Ambiguität dieser Kriegslyrik hin, die Eisler nicht geheuer sein konnte: Klabund stimmte bekanntlich in den Chor der Begeisterung zum Kriegsausbruch mit ein, neunmal meldete er sich freiwillig zum Kriegsdienst, neunmal wurde er aufgrund eines Lungenleidens abgewiesen und war darüber fürchterlich enttäuscht - eine Enttäuschung, der er sogar in einem Gedicht unter dem Titel Landsturm ohne Waffe weinerlichen Ausdruck verlieh. ${ }^{7}$ Um dennoch das Seine zum Krieg beizutragen, sammelte und verfasste er Soldatenlieder von tumber Volkstümlichkeit, die mit ihrem Offiziersgehabe den Patriotismus, den Heldenmut und das Durchhaltevermögen der deutschen Truppen stärken sollten. So etwa das Lied der Kriegsfreiwilligen:

\author{
Brüder, laßt uns Arm in Arm \\ In den Kampf marschieren! \\ Schlägt der Trommler schon Alarm \\ Fremdesten Quartieren. \\ West- und östlich glüht der Brand, \\ Sternenschrift im Dunkeln \\ Läßt die Worte funkeln: \\ Freies deutsches Land!
}

7 Vgl. Kuei-Fen Pan-Hsu:Die Bedeutung der chinesischen Literatur in den Werken Klabunds. Eine Untersuchung zur Entstehung der Nachdichtungen und deren Stellung im Gesamtwerk, Frankfurt am Main: Peter Lang, 1990, ab S. 84. 
Hebt die Hand empor:

Kriegsfreiwillige vor! ${ }^{8}$

Eine Lyrik, die den Krieg verherrlicht, muss sich jedoch nicht auf dieses burschenhafte Valleri beschränken. Das Leiden der getrennten Geliebten, die Entbehrungen im Dienst, der körperliche Schmerz der Verwundung, die Trauer um die Gestorbenen, die Ohnmacht vor den feindlichen Angriffen und selbst die Formen der seelischen Verhärtung, der Resignation und des Zynismus, ohne die der Kriegsbetrieb gar nicht auszuhalten wäre - all diese Erfahrungen der Negativität fehlen auch in jener Lyrik nicht, die den Krieg affirmiert. Denn die kriegerischen Tugenden, die solche Lyrik besingt, sind ja nur im Widerstand gegen die Negativität recht zu begreifen: Tapferkeit und Heldenmut, Härte und Ausdauer, Opferbereitschaft und Vaterlandsliebe beweisen sich erst am Negativen, das sie überwinden - je größer die Not, desto größer die Tat.

So sterben die tapferen Jünglinge in Klabunds Soldatenliedern auf dem Schlachtfeld so, wie die Sterne fallen, ${ }^{9}$ und aus ihrem Körper fließt das Blut »wie Gold «, die stolzen Dragoner und Husaren fallen zerschossen dumpf von ihren stolzen Pferden, »aus tausendfach vergossnem Blut / Spriesst junges Grün an Weiden und an Ebereschen $«{ }^{10}{ }^{0}$ Die Nachdichtungen chinesischer Kriegslyrik schreiben sich in diese Logik ein. Noch der resignierte Zug in den Gedichten von Li Bai, die das Elend des Winterkriegs und die Sinnlosigkeit seiner Opfer beklagen, lässt sich als Soldatentugend verstehen: Die Haltung, mit allem durch zu sein, nichts mehr zu hoffen, von keinem Elend mehr geschreckt zu werden und zuletzt der Sinnlosigkeit des Kriegs ins Gesicht zu lachen - dieser resignierte Gleichmut, der auch im Umfeld des deutschen Expressionismus gerne mit dem fernen Osten assoziiert wurde, ${ }^{11}$ macht den Soldaten im Grabenkrieg erst überlebensfähig. Die Negativität des Kriegs sich gänzlich vor Augen zu stellen, ist ein bewährtes Mittel, um die Größe der Kriegstaten und die Standhaftigkeit der Helden zu besingen.

Auch in Klabunds späteren Gedichten aus der Himmelsleiter treten die Bilder des Grauens in den Dienst am Kriegsmythos. In ihnen weicht der frühere volkstümliche Ton einer gewissen Eindringlichkeit, welche die Nähe zum Expressionismus sucht. So in Klabunds Gewitternacht, in der die expressionistische Sehnsucht nach dem Weltenbrand mit jener Naturalisierung des Grabenkriegs einhergeht, die Ernst Jünger ${ }^{12}$ bald darauf ausschlachten wird:

Zitiert nach der Transkription der Originalausgabe unter http://klabund.eu/wp/drago ner-und-husaren/.

9 Es fällt ein Stern.

10 Frühling 1915, zitiert nach der Transkription der Originalausgabe unter http://klabund.eu/ wp/die-himmelsleiter/.

11 Vgl. Ming Jian: Expressionistische Nachdichtungen chinesischer Lyrik, Frankfurt am Main: Peter Lang, 1990.

Vgl. Ernst Jünger: In Stahlgewittern, Stuttgart: Klett-Cotta, 2013. 
Und immer mehr und immer fort

Und Rausch und Blut und Sang und Mord,

Wir sterben, sterben, sterben.

Der Himmel donnert, Wolke kracht,

Ein Blitz knallt nieder durch die Nacht

Und schmeisst die Welt in Scherben. ${ }^{13}$

Die Visionen von Gewalt und Zerstörung, wie man sie bekanntlich auch bei Expressionisten wie Georg Heym oder dem Futuristen Filippo Marinetti findet, sind keineswegs im Sinne einer bloßen Ablehnung des Kriegs zu deuten. Sinn und Funktion solcher Bilder sind nicht einfach zu bestimmen - sind es Gewaltfantasien, die es dem kleinbürgerlichen Leser erlauben, sich im Schein mit jenen Mächten zu identifizieren, denen er im wirklichen Leben unterliegt? Wird in ihnen die Negativität der Zerstörung als solche gefeiert? Sind es Reizmittel, mit deren Hilfe man dem unwirklichen Grau der Gewohnheit entflieht, um den Schauer einer abgründigen Wirklichkeit zu erleben? Oder sind es Schreckensbilder, welche das Grauen bannen sollen, das sie evozieren? Sind es Wunschbilder, in denen die Katastrophe als reinigende Kraft herbeigesehnt wird, welche die Welt verjüngen und von der Krankheit Moderne kurieren soll? Wie immer man die Negativität affirmativer Kriegslyrik deutet, was sie in jedem Fall leistet, ist eine Überhöhung und Mystifizierung des faktischen Leidens, eine Erhebung des stupiden Kriegselends in eine Sphäre größerer Zusammenhänge, in eine Region höheren Sinns, an dem man - im Jargon jener Zeit gesprochen - teilhaben kann.

In ausdrucksvolle, eindringliche Verse gebracht, verleiht so noch die niedrigste Gewalttat dem Krieg einen schauerlichen Glanz. Die künstlerische Konsequenz aus dieser Situation zog bekanntlich Dada: Die radikale Zurückweisung des Kriegs geht bei den Dadaisten mit einem refus des Sinnvollen überhaupt einher, der Vermeidung jeglicher dichterischer Kohärenz, die noch in der Darstellung von Leid und Trauer die zerfetzte Wirklichkeit zusammenflicken würde. Daraus wird die Frontstellung der Dadaisten gegen den Expressionismus verständlich, wie sie sich schon in den Aktionen des Zürcher Dada abzeichnet, und die im Berliner Dada-Manifest von 1918 explizit wird:

Die höchste Kunst wird diejenige sein, die in ihren Bewußtseinsinhalten die tausendfachen Probleme der Zeit präsentiert, der man anmerkt, daß sie sich von den Explosionen der letzten Woche werfen ließ, die ihre Glieder immer wieder unter dem Stoß des letzten Tages zusammensucht. Die besten und unerhörtesten Künstler werden diejenigen sein, die stündlich die Fetzen ihres Leibes aus dem Wirrsal der Lebenskatarakte zusammenreißen, verbissen in den Intellekt der

13 Zitiert nach der Transkription der Originalausgabe unter http://klabund.eu/wp/die-himmels leiter/. 
Zeit, blutend an Händen und Herzen. Hat der Expressionismus unsere Erwartungen auf eine solche Kunst erfüllt, die eine Ballotage unserer vitalsten Angelegenheiten ist? Nein! Nein! Nein! ${ }^{14}$

Dass noch die Negativität expressionistischer Kriegslyrik sich in Affirmation verkehren kann, gilt auch für die Musik. Es ist daher naiv, wenn Stefan Hanheide jeglichen Ausdruck von Gewalt und Leiden in Kunstliedern aus der Zeit des Ersten Weltkriegs, etwa von Richard Strauss (1864-1949), Paul Hindemith (1895-1963), Charles Ives (1874-1954) oder Franz Léhar (1870-1948), als Zeichen einer »kritischen Stellungnahme zum Krieg « ${ }^{15}$ deutet - und die Zurückhaltung der Künstler in ihrer Kritik am Krieg mit dem Umstand möglicher Strafverfolgung und Zensur entschuldigt. Schon der Ausdruck des >Tragischen<, der nach Hanheide in der Musik vernehmbar wird, zeigt eine Ambivalenz an, die er nicht wahrhaben will: Tragisch ist das unvermeidbare Umschlagen des Guten ins Schlechte, die Unabwendbarkeit eines Leidens, das den tragischen Helden dadurch, dass er es flieht, nur umso sicherer ereilt. Die industriell belieferte Materialschlacht des Ersten Weltkriegs wird daher völlig verzeichnet, wenn man sie als Tragödie darstellt: Weder ist das Leiden der Soldaten unvermeidbar noch spielt ihr individuelles Handeln für den Verlauf des Kriegs wie für das eigene Überleben irgendeine Rolle. Es gibt weder Tragik noch tragisches Heldentum im modernen Krieg; die Klage um den Gefallenen als tragisches Opfer ist daher eine Form der Verklärung.

\section{Kriegsmüdigkeit}

Mit dem Andauern des Kriegs verschlechterte sich erwartungsgemäß selbst die Stimmung der feurigsten Patrioten: ${ }^{16}$ Ermattung und Überdruss am Krieg macht sich auch bei Klabund breit, die Sehnsucht nach dem Leben im Frieden verdrängt den Heldenmut. Dieser Gesinnungswandel kommt in der zweiten Nachdichtung Klabunds zum Ausdruck, die Eisler für sein Anti-Kriegs-Oratorium vertonte:

\section{Der müde Soldat}

Ein kahles Mädchen. Heckenblaßentlaubt. Sie steht am Weg. Ich gehe weit vorbei.

14 »Dadaistisches Manifest«, zit. nach: Manifeste und Proklamationen der europäischen Avantgarde 1909-1938, hg. von Wolfgang Asholt und Walter Fähnders, Stuttgart: Metzler, 2005, S. 145.

15 Stefan Hanheide: Wahrnehmungen von Kriegstragik im Kunstlied während des Ersten Weltkriegs, in: Musik bezieht Stellung. Funktionalisierungen der Musik im Ersten Weltkrieg, hg. von Stefan Hanheide, Dietrich Helms, Claudia Glunz und Thomas F. Schneider, Göttingen: Universitätsverlag Osnabrück, 2013, S. 307-332, hier S. 312.

16 Zu Klabunds Verhältnis zum Krieg vgl. Pan-Hsu: Die Bedeutung der chinesischen Literatur in den Werken Klabunds, S. 87. 
So stehen alle: Reih in Reih, Und Haupt an Haupt.

Was weiß ich noch von heiligen Gewässern

Und von des Dorfes Abendrot?

Ich bin gespickt [bei Eisler: behängt] mit tausend Messern

Und müde von dem vielen Tod.

Der Kinder Augen sind wie goldner Regen,

In ihren Händen glüht die Schale Wein.

Ich will mich unter Bäumen schlafen legen

Und kein Soldat mehr sein. ${ }^{17}$

Klabund bearbeitet in dieser Übertragung aus dem Buch der Lieder (Shi Jing 詩經) das Motiv der Kriegsmüdigkeit. Sie bezeichnet ein Schwinden der Kräfte, ein Verlieren des Durchhaltevermögens, das mit einer Sehnsucht nach dem Frieden einhergeht. Nach all den Jahren der Entbehrung, der Aufopferung in Reih und Glied, erschlafft der Wille des Kämpfers, der Soldat lässt sich gehen. Karl Kraus hat diesen Pazifismus aus Überdruss treffend charakterisiert:

Kriegsmüde - das ist das dümmste von allen Worten, die die Zeit hat. Kriegsmüde sein das heißt müde sein des Mordes, müde des Raubes, müde der Lüge, müde der Dummheit, müde des Hungers, müde der Krankheit, müde des Schmutzes, müde des Chaos. War man je zu all dem frisch und munter? So wäre Kriegsmüdigkeit wahrlich ein Zustand, der keine Rettung verdient. Kriegsmüde hat man immer zu sein, das heißt, nicht nachdem, sondern ehe man den Krieg begonnen hat. Aus Kriegsmüdigkeit werde der Krieg nicht beendet, sondern unterlassen. Staaten, die im vierten Jahr der Kriegführung kriegsmüde sind, haben nichts Besseres verdient als - durchhalten! ${ }^{18}$

Kriegsmüdigkeit setzt Kriegsmunterkeit voraus, von der sie abfällt. Was Kraus in seinen Streifzügen durch die publizistische Unvernunft vermerkt, trifft auch auf Klabunds Dichtungen zu. Tendieren sie gegen Ende des Weltkriegs hin zur Desillusion, so ist diese dialektisch an eine vormalige Begeisterung gebunden, von der sie daher nie ganz frei wird - die Enttäuschung, die in der Kriegsmüdigkeit zum Ausdruck kommt, ist in erster Linie eine über die eigene Kraftlosigkeit - und den ausbleibenden Sieg. Solcher Pazifismus ist daher ebenso unstet wie der Enthusiasmus des Augusterlebnisses: Sobald der Sieg wieder in die Nähe rückt, verfliegt die Mümeln-und-berauschtes-gong/.

Karl Kraus: Kriegsmüde, in: Die Fackel 20 (1918), Nr. 474-483, S. 153. 
digkeit. Obwohl Klabund die Lektüre der chinesischen Kriegslyrik im Nachhinein als Erlebnis eines Gesinnungswandels hin zum Pazifismus inszenierte, wird er sich auch danach wieder - erfolglos - als Freiwilliger zum Kriegsdienst melden. ${ }^{19}$

\section{Der Widerspruch des Pazifismus}

Unabhängig von den Irrungen Klabunds wird die Selbstdistanz, die ich in Eislers Vertonung von $\mathrm{Li}$ Bais Gedicht zu vernehmen glaube, mit der Dialektik von affirmativen und negativen Momenten in der Kriegslyrik zusammenhängen. Bisher kam jedoch sozusagen erst die eine Seite der Dialektik in den Blick - die negative Dimension affirmativer Kriegslyrik. Die andere Seite bildet die Position, die Eisler gesucht haben wird: die Haltung gegen den Krieg - der Pazifismus. Denn auch diese Position ist nicht frei von Widersprüchen. In diesem Sinne notiert Eisler 1921 spöttisch: »Wenn man den Pacifisten Krieg erklärt, so sagen sie: Pardon bitte, das ist ein Irrtum. $\ll^{20}$

Der Pazifist glaubt eine Position jenseits gewalttätiger Konflikte einnehmen zu können: Er hält Gewalt für eine Gesinnungsfrage, der man sich durch Umsinnen entziehen könne. Wer ihm mit Gewalt kommt, den verweist er an die Nebentür - als Pazifist hätte er damit nichts am Hut. Der Witz will den Pazifismus als Scheinposition entblößen: Sie lasse sich realiter gar nicht einnehmen.

Dieser Einwand gegen den Pazifismus wurde in den 1960er Jahren vom libertären Philosophen Jan Narveson auf den Begriff gebracht: ${ }^{21}$ Der Pazifismus widerspreche sich selbst. Unter Pazifismus versteht Narveson eine Position, die jedem Menschen das Recht auf physische Unversehrtheit zuspricht, die unter keinen Umständen, also auch durch keine anderen Rechtsgründe aufgehoben werden kann. Der Einsatz von Gewalt kann daher durch keinen Zweck gerechtfertigt werden. Der Begriff des Rechts aber impliziert, so Narvesons Argument, Gewalt als letztes Mittel der Rechtsgarantie: Was auch immer jemandem durch ein Recht zugesprochen wird, mit diesem Recht erhält er auch das Recht, dass dieses Recht gegen Verletzung geschützt respektive seine Übertretung bestraft wird - wenn nötig, mit Gewalt. Denn ein Recht, dessen Missachtung keinerlei Konsequenzen hätte, wäre kein Recht: Recht ist, in letzter Instanz, immer gewaltbewehrtes Recht. Daher gerät der Pazifist in einen Widerspruch: Er lehnt Gewalt auch als Mittel gegen die Überschreitung des Gewaltverbots ab. Damit wird das Verbot aber de facto inexistent. Das unbedingte Recht auf physische Unversehrtheit hebt sich selbst auf:

20 Hanns Eisler: [Wiener Tagebuch 1921-1922], in: Hanns Eisler. Gesammelte Schriften 1921-1935, hg. von Tobias Faßhauer und Günter Mayer, Wiesbaden: Breitkopf \& Härtel, 2007, S. 1-22, hier S. 14.

Jan Narveson: Pacifism. A Philosophical Analysis, in: Ethics 75 (1965), S. 259-271. 
What this all adds up to, then, is that if we have any rights at all, we have a right to use force to prevent the deprivation of the thing to which we are said to have a right. But the pacifist, of all people, is the one most concerned to insist that we do have some rights, namely, the right not to have violence done to us. This is logically implied in asserting it to be a duty on everyone's part to avoid violence. And this is why the pacifist's position is self-contradictory. In saying that violence is wrong, one is at the same time saying that people have a right to its prevention, by force if necessary. Whether and to what extent it may be necessary is a question of fact, but, since it is a question of fact only, the moral right to use force on some possible occasions is established. $^{22}$

Narvesons Argumentation ist offensichtlich tendenziös: Der Pazifismus, der im Kontext der Veröffentlichung seines Texts infrage steht, der Pazifismus der Vietnamkrieg-Proteste, ist natürlich nicht jener bedingungslose Pazifismus, gegen den Narveson argumentiert. Kaum ein Kriegsgegner will den Rechtsstaat abschaffen, nur weil dieser Gewalt in Form gerichtlich entschiedener Sanktionen anwendet. Dennoch ist Narvesons Argument aufschlussreich. Es hilft, die Begründung einer Opposition gegen den Krieg zu schärfen. Narveson zeigt, dass diese Ablehnung des Kriegs nicht auf der Ablehnung von Gewalt als solcher beruhen kann, solange sie am Begriff des Rechts orientiert ist. Es gibt für den Pazifisten also mehrere Auswege: Er kann das Gewaltverbot einschränken, um Raum für rechtmäßige Gewalt zu schaffen. Damit verwandelt sich die Frage des Pazifismus in die Frage des gerechten oder zumindest gerechtfertigten Kriegs: das jus ad bellum. Auf dieser Ebene findet normalerweise der Protest gegen Krieg statt: Was von den Machthabern als legitimer Kriegsgrund vorgeschoben wird - für gewöhnlich Selbstverteidigung und Friedenssicherung -, stellt sich meist als blanker Betrug heraus, hinter dem sich unschwer Partikularinteressen erkennen lassen. Oder man verbindet den Pazifismus mit einer Kritik des Rechts als solchem und setzt die Gewaltlosigkeit als eine Forderung jenseits oder diesseits von strafbewehrten Rechtsverhältnissen.

\section{Vom Krieg zum Kampf}

Die Position, die Hanns Eisler im Verlauf der 1920er Jahre entwickeln wird, kritisiert den Krieg als ungerechtfertigte Gewalt. Sie fußt auf der Unterscheidung zweier Formen politischer Gewalt: Krieg und Kampf. Krieg bezeichnet die illegitime Form der Gewalt, der Massenbetrug des zwischenstaatlichen Konflikts - Kampf hingegen bezeichnet die gerechtfertigte, revolutionäre Gewalt, die auf die Überwindung der Klassenherrschaft zielt. Was immer man von Eislers sozialistischen Position halten 
mag: Man muss sich den Zusammenhang dieser Ideen in Erinnerung rufen, will man seine ästhetischen Entscheidungen nachvollziehen. 1931 ruft Eisler in diesem Sinne zum Widerstand gegen die nationalsozialistischen Kriegstreiber auf:

Um bei den jungen Leuten, die bei den Faschisten stehen oder mit ihnen sympathisieren, die »Kriegsideologie« zu bekämpfen, die sie als Schlachtvieh des internationalen Kapitals gegen die Sowjetunion zu treiben droht, müßte man eine spezielle ideologische Campagne gegen die romantische Kriegsauffassung führen. Wenn, wie der junge Faschist behauptet, erst im Kriege der Mann entsteht, so ist darauf hinzuweisen, daß der Klassiker der preußischen Kriegstheorie, General Clausewitz, seine berühmte Formulierung des Krieges als »die Fortsetzung der Politik mit anderen Mitteln« schon vor 100 Jahren aufstellte. Ein neuer Krieg der Bourgeoisie würde die Unterdrückung und Ausbeutung nur verstärken, er ist also nicht als geheimnisvolles nationales Blut-Phänomen, sondern als Transferierung des Ausbeutungs-Objekts vom laufenden Band in die Materialschlacht anzusehen. ${ }^{23}$

Der Krieg ist für Eisler immer eine Verlängerung der Klassenherrschaft, in ihm werden die Ausgebeuteten unter dem Vorwand von Ehre, Patriotismus und Friedenssicherung zum Vorteil der Bourgeoisie an die Schlachtbank geführt: Auf beiden Seiten der Gefechtslinie steht dieselbe unterdrückte Klasse. Unter dem Kommando ihrer Unterdrücker bekämpft sie sich selbst, statt sich gegen ihre Kommandeure aufzulehnen. Dieser immer wiederkehrende Betrug bringt Eisler musikalisch in den Chor-Variationen Gegen den Krieg von 1936 zum Ausdruck, die unablässig um eine chromatisch gestufte Zwölftonreihe wie um eine historische Invariante kreisen. Auch der Text Bertolt Brechts verharrt auf ein und demselben Gedanken: »Dieser Krieg ist nicht unser Krieg« heißt es in der Fuge (20.-24. Variation); »Wer da vom Feind spricht, ist unser Feind « (14. Variation); "Wenn die Ob’ren von Frieden reden, weiß das gemeine Volk, dass es Krieg gibt« (9. Variation); »Wenn die Ob’ren von Frieden sprechen, Mann auf der Straße, lass alle Hoffnung fahren. Wenn die Ob'ren Nichtangriffspakte schließen, kleiner Mann, mach dein Testament« (3.-4. Variation); »Sie reden wieder von großen Zeiten / von Ehre / von Siegen, Marie, weine nicht « (11.-13. Variation).

Gegen den Krieg bringen Eisler und Brecht nicht die Gewaltlosigkeit, sondern eine andere, legitime Gewalt in Stellung. Der Pazifismus - die Kriegsgegnerschaft teilt sich somit in zwei entgegengesetzte Positionen: Der bürgerliche Pazifismus auf der einen Seite, der die Gewalt der Klassenherrschaft verleugnet, und die revolutionäre Ablehnung des Kriegs auf der anderen Seite, die in ihm die Verlängerung der Klassenherrschaft erblickt, die es - wenn nötig gewaltsam - zu bekämpfen gilt. In 
solcher Perspektive ist es kein Widerspruch, wenn der Kriegsgegner Eisler die Musik zur »Waffe im Klassenkampf $\aleph^{24}$ macht:

Die Musik hat, genau so wie jede andere Kunst einen bestimmten gesellschaftlichen Zweck. In der Bourgeoisie ist der Zweck, das ist die Funktion der Musik, eine scheinbare Zwecklosigkeit. Musik wird von der bürgerlichen Gesellschaft vor allem als Erholung zur Reproduktion der Arbeitskraft benutzt. [...] Die Arbeitermusikbewegung muss sich über die neue Funktion der Musik, das sind: Aktivierung zum Kampf und politische Schulung klar werden. [...] Die Aufgabe der Arbeitermusik wird es sein, die Sentimentalität, den Schwulst aus der Musik zu liquidieren, da diese Empfindungen vom Klassenkampf ablenken. Das wichtigste Prinzip der Kampfmusik ist, dass wir die Musik einteilen in solche, welche selbst musiziert werden soll, also Kampflieder, satirische Lieder, etc. und solche, welche angehört werden soll. ${ }^{25}$

Die Liquidation des Sentimentalen, die »scharfe, kalte Grundhaltung ${ }^{26}$ der Musik, die Eisler vorschwebt, kann in zwei Richtungen ausgelegt werden. Zum einen wendet sich Eisler gegen Ende der 1920er Jahre bekanntlich von den `Nachkriegsexperimenten ‘ der musikalischen Avantgarde ab, der er selbst zugehört: Weil die musikalische Ausbildung ein Klassenprivileg sei, könne die Arbeitermusik nicht unmittelbar die avanciertesten Techniken der bürgerlichen Musik übernehmen. Die Kampfmusik muss die Verfeinerungen der bürgerlichen Ausdruckskunst aufgeben und Vereinfachungen vornehmen: Sentimentalität steht hier für die verfeinerte Reflexion, das Zweifelnde und Komplexe. Andererseits richtet sich die Rede vom Schwulst auch gegen die Anbiederung der Kampfmusik ans Bekömmliche: den >roten Schlager, das Tendenzlied, die tradierten Arbeiterchöre, welche ein veraltetes Idiom der bürgerlichen Musik des 19. Jahrhunderts übernehmen und ihnen sozialistische Texte überstülpen. Das Sentimentale steht dann, gerade umgekehrt, für das aufgesetzt Naive und Rührselige, das Sich-Dumm-Stellen des Volkstümlichen. ${ }^{27}$ Beiden hält Eisler eine gewisse martialische Schärfe, eine neusachliche Kälte ${ }^{28}$ entgegen, welche die Entschlossenheit zum Klassenkampf stärken soll: militärische Tugenden also, die nun gegen den Krieg gewendet werden sollen.

24 Hanns Eisler: Neue Methoden der Kampfmusik, in: Hanns Eisler. Gesammelte Schriften 19211935, hg. von Tobias Faßhauer und Günter Mayer, Wiesbaden: Breitkopf \& Härtel, 2007, S. 156.

25 Ebd., S. $155 \mathrm{f}$.

26 Ebd., S. 156.

27 Vgl. Hanns Eisler: Die Erbauer einer neuen Musikkultur, in: Hanns Eisler. Gesammelte Schriften 1921-1935, hg. von Tobias Faßhauer und Günter Mayer, Wiesbaden: Breitkopf \& Härtel, 2007, S. 132-151.

28 Vgl. eine Kulturgeschichte dieses Charakters mit Bezug auf Brecht: Helmut Lethen: Verhaltenslehren der Kälte. Lebensversuche zwischen den Kriegen, Frankfurt am Main: Suhrkamp, 2014. 
Mit dem Aufruf zur Liquidation des Sentimentalen - der Text entsteht bezeichnenderweise im Umfeld der Komposition zu Brechts Maßnahme - macht Eisler jene widersprüchliche Bewegung der Selbstdistanzierung, ein Selbstmisstrauen explizit, die seine Kompositionen seit der frühen Vertonung des Gedichts nach Li Bai prägt. Die martialische Kälte richtet sich gegen die falsche Gefühlswärme jener Ausdruckskunst, der doch letztlich auch Eislers Lieder zugehören. Seine Fähigkeit zum differenzierten Ausdruck wird ihm selbst unerträglich, weil eine solche Differenziertheit dem individuellen Ausdruck eine Wichtigkeit zumisst, die er in einer Welt verdinglichter Verhältnisse nicht besitzt: Das Kunstlied als solches ist lächerlich geworden. ${ }^{29}$ Deshalb unterbricht sich Eislers Musik durch den Einsatz des Kunstlosen, ist „Vulgär aus Geschmack « ${ }^{30}$ und verspottet sich selbst, wie etwa ganz deutlich im Schweyk.

Aber auch die Sphäre des Kollektiven, die Eisler dem bürgerlichen Individualismus entgegensetzt, der Gleichschritt des Marschs, die Schmähreime im Schlagerton, die Hetze des Kampflieds bleiben nicht intakt: Ihre Simplifizierungen sind auch musikalisch Selbsttäuschungen, die Eisler - zugegebenermaßen nicht immer - unterbricht. Erst wo sich diese beiden Sphären in unauflöslicher Spannung aneinander aufreiben, sind seine Werke am überzeugendsten: in den Männerchören op. 13, deren spöttischer Vorspruch die echte Verzweiflung des darauf folgenden Gesangs der Besiegten untergräbt, der wiederum nur durch einen Ruck ins zuversichtliche Ende geführt werden kann mit den Worten »Nicht verzagen, unser Sieg wird der letzte sein«; oder in der Bühnenmusik zu Brechts Die Mutter, deren Chöre durch den kargen Satz der Instrumente, das Schäbige von Banjo, Trommel und Klarinette und das stimmungsbrechende Prinzip der Montage gestört werden. Das oft gegen Eisler geführte Argument, der martialische Charakter seiner Musik unterscheide sich vom Militarismus der politischen Reaktion nur durch den Text, ${ }^{31}$ kann hier wie in vielen seiner Kampflieder nicht überzeugen - was vielleicht auf eine bloße Melodie zutrifft, gilt nicht für die konkrete Faktur eines Lieds in seiner Gänze: Ihr brüchiger Charakter macht diese Kampfmusik für den Faschismus unbrauchbar - und den sozialistischen Parteidiktaturen suspekt.

\section{Das Ende der Revolution}

Mit großer Klarheit hat Carl Dahlhaus den Widerspruch erfasst, an dem sich Eislers funktionale Musik abarbeitet:

Politische Musik wäre, wenn sie wirkungslos bliebe, eine bloße Attrappe ihrer selbst, in schlimmen Fällen ein durch Überflüssigkeit Zeitungsausschnitte, in: Adorno. Gesammelte Schriften, Bd. 18, hg. von RolfTiedemann, Frankfurt am Main: Suhrkamp, 1984, S. 324-327.

30 Adorno: Notizen über Eisler, S. 77.

31 Vgl. Reinhold Brinkmann: Musikalische Maßnahmen Eislers, in: Über Musik und Politik, hg. von Rudolf Stephan, Mainz: Schott, 1971, S. 9-23. 
jämmerliches sacrificium intellectus. Um aber den Zweck zu erreichen, auf den sie zielt, muß sie sich den musikalischen Gewohnheiten und Vorurteilen der Hörer angleichen, in deren politisch-soziales Bewußtsein sie verändernd eingreifen soll. [...] Die politische Emanzipation, die gemeint ist, erschöpft sich jedoch nicht in sich selbst, sondern stellt ein Teilmoment einer allgemeinen Emanzipation dar, die auch die Musik umfaßt. Und umgekehrt ist nach marxistischer Überzeugung die schlechte und dumme Musik, die von allen Seiten auf uns eindringt und der sich kaum jemand entziehen kann, Produkt, Ausdruck und Kitt einer miserabel eingerichteten Gesellschaft. [...] Der engagierte Komponist, der politisch zu wirken versucht, benutzt also musikalische Mittel, deren Aufhebung durch Überflüssig-Werden das Ziel ist, dem er eigentlich - in letzter Instanz - zustrebt. Um den bestehenden Zustand zu verändern, fühlt er sich gezwungen, an den musikalischen Schwachsinn anzuknüpfen, dessen Absterben er - als Folge oder Teilmoment einer geglückten Revolution - erhofft. ${ }^{32}$

Die Musik der Revolution steht im Widerspruch zur Revolution der Musik: Die politisch wirksame Musik bedient sich der Mittel, deren Abschaffung sie anstrebt. Dieser Widerspruch, den Dahlhaus im Verhältnis von Kunst und Politik verortet, liegt in Wahrheit im Gedanken der sozialistischen Revolution selbst, ${ }^{33}$ denn diese Revolution soll in eine Übergangszeit führen - die Diktatur des Proletariats -, während der sich die revolutionäre Klasse vorrevolutionärer Herrschaftsmittel bedient, um das eigentliche Ziel der Revolution - die herrschaftsfreie Gesellschaft - herbeizuführen. Die Herrschaft des Proletariats zielt auf die Abschaffung der Klassenherrschaft überhaupt, also auch auf ihre Selbstabschaffung. Der revolutionäre Staat verwirklicht sich in der Abschaffung von Staatsgewalt: Er erfüllt sein Ziel, indem er abstirbt und sich verwandelt »in etwas, was eigentlich kein Staat mehr ist.$^{34}$

Folgt man Eislers Überlegungen, so gilt dasselbe für die revolutionäre Musik. Ihre Aneignung der bürgerlichen Kunst zielt nicht auf die Errichtung einer proletarischen Kultur, sie will keine Musik der Arbeiter etablieren. Sie ergreift vielmehr die Ausdrucksformen der herrschenden wie der unterdrückten Klassen, um sie so umzuformen und aneinander abarbeiten zu lassen, dass sie ihren gegensätzlichen Charakter verlieren und sich zuletzt selbst aufheben. Was in der klassenlosen Gesellschaft an ihre Stelle treten wird, kann nicht antizipiert werden. Der Charakter des Von-sich-Abstand-Nehmens, der Eislers Musik eigen ist und sich als ihr produktiver Grundwiderspruch erweist,

32 Carl Dahlhaus: Thesen über engagierte Musik, in: Musik zwischen Engagement und Kunst, hg. von Otto Kolleritsch, Graz: Universal Edition, 1972 (Studien zur Wertungsforschung, Bd. 3), S. 7-19, hier S. 8 f.

33 Vgl. Gunnar Hindrichs: Philosophie der Revolution, Berlin: Suhrkamp, 2017, S. 267-272.

34 Vgl. Wladimir Iljitsch Lenin: Staat und Revolution. Die Lehre des Marxismus vom Staat und die Aufgaben des Proletariats in der Revolution, in: Lenin. Werke, Bd. 25, Berlin: Dietz, 1968, S. 393-507, hier S. 432. 
kann daher als ihr revolutionärer Zug gedeutet werden: Als revolutionäre Musik kann sie sich nicht selbst affirmieren, ohne ihre Sache zu betrügen.

Die sozialistische Revolution von 1917 hat freilich ein anderes Ende genommen. Sobald sich der revolutionäre Staat zur Parteidiktatur verfestigt, die das Ziel ihrer Selbstabschaffung verleugnet, die klassenlose Gesellschaft per Dekret verkündet und entsprechende künstlerische Ausdrucksformen anbefiehlt, kann von einer revolutionären Kunst keine Rede mehr sein. Eine solche Verfestigung ist auch in Eislers Musik festzustellen - der Geheime Aufmarsch, das Einheitsfrontlied, die Deutsche Symphonie, die Deutschen Volklieder und die Hymne der DDR sind nur die deutlichsten Fälle einer nicht mehr revolutionären Musik. Das drängende, sich selbst überstürzende »Vorwärts! « des Solidaritätslieds ist in ihr längst verklungen.

In seiner späten Komposition zu Brechts Bilder aus der Kriegsfibel unternimmt Eisler abermals den Versuch, eine Anti-Kriegskantate zu komponieren. Die Lieder sollen zu den projizierten Fotografien aus der amerikanischen Kriegsberichterstattung gespielt werden, welche spektakulär die Zerstörungen des Zweiten Weltkriegs zeigen und zu denen Brecht seine seltsam plakativen Gedichtkommentare schrieb. Die unangenehme Verdopplung des Bildsinns wiederholt sich in Eislers Miniaturen, die sich nur durch ihre extreme Kürze vor ihrer eigenen Plattheit retten. Der Epilog nimmt das Revolutionswort Lenins auf: »Und lernt das Lernen und verlernt es nie! «; aber in der infantilisierten Weise, die ihm Eisler unterlegt, verkommt der revolutionäre Appell zum pedantischen Merkspruch, der sich mit erhobenem Finger an jene richtet, die »jetzt hier sitzen« können. Man könnte versuchen auch hierin eine Geste der Selbstdistanz zu lesen: eine Ausstellung des Lächerlichen im revolutionären Anti-Kriegspathos - aber die Umdeutung will nicht recht gelingen. Das vorzeitige Abreißen der Nummern, das dieses seltsame Alterswerk auszeichnet, führt uns jedoch zur frühen Vertonung der Roten und weißen Rose zurück. Auch sie ließ den musikalischen Schein jäh abreißen, zeigte sie doch die Szene einer Selbsttäuschung, die in sich zusammenfällt, ein von der Hoffnung hervorgetriebenes Wunschbild das von der Wirklichkeit enttäuscht werden muss. In dieser Enttäuschung zeigt sich die Intelligenz, die den Schein, in den sie sich hineinsteigert, zu durchschauen vermag. Aber diese Intelligenz, das desillusionierte Bewusstsein, wäre doch ohne den Schein, den sie durchschaut, ebenso nichtig wie der Schein, den sie zerreißt - der zerrissene Schein ist mehr als die bloße Scheinlosigkeit. In dieser Angewiesenheit der Intelligenz auf die Illusion der Hoffnung steckt so etwas wie der Wahrheitsgehalt von Eislers Musik: In ihr zeigt sich Wahrheit als Selbstenttäuschung.

\section{Literatur}

Adorno, Theodor W.: Notizen über Eisler [1992], in: Hanns Eisler, hg. von Albrecht Dümling, Frankfurt am Main: Stroemfeld, 2010 (Querstand. Musikalische Konzepte, Bd. 5/6), S. 73-83.

Adorno, Theodor W.: Eisler. Zeitungsausschnitte, in: Adorno. Gesammelte Schriften, Bd. 18, hg. von Rolf Tiedemann, Frankfurt am Main: Suhrkamp, 1984, S. 324-327. 
Asholt, Wolfgang/Fähnders, Walter (Hg.): Manifeste und Proklamationen der europäischen Avantgarde 1909-1938, Stuttgart: Metzler, 2005, https://doi.org/10.1007/978-3-47600104-7.

Bethge, Hans: Die chinesische Flöte, Leipzig: Insel-Verlag, 1907.

Brinkmann, Reinhold: Musikalische Maßnahmen Eislers, in: Über Musik und Politik, hg. von Rudolf Stephan, Mainz: Schott, 1971, S. 9-23.

Dahlhaus, Carl: Thesen über engagierte Musik, in: Musik zwischen Engagement und Kunst, hg. von Otto Kolleritsch, Graz: Universal Edition, 1972 (Studien zur Wertungsforschung, Bd. 3), S. 7-19.

Eisler, Hanns: Bilder aus der Kriegsfibel, in: Lieder und Kantaten, Bd. 10, Leipzig: VEB Breitkopf \& Härtel, 1963, S. 31-60.

Eisler, Hanns: Die Erbauer einer neuen Musikkultur, in: Hanns Eisler. Gesammelte Schriften 1921-1935, hg. von Tobias Faßhauer und Günter Mayer, Wiesbaden: Breitkopf \& Härtel, 2007, S. 132-151.

Eisler, Hanns: Gegen den Krieg, in: Lieder und Kantaten, Bd. 3, Leipzig: VEB Breitkopf \& Härtel, 1958, S. 225-236.

Eisler, Hanns: Kämpft um die schwankenden Massen! Wie gewinnen wir Kleinbürger und junge Leute? [1931], in: Hanns Eisler. Gesammelte Schriften 1921-1935, hg. von Tobias Faßhauer und Günter Mayer, Wiesbaden: Breitkopf \& Härtel, 2007, S. 105 f.

Eisler, Hanns: Neue Methoden der Kampfmusik, in: Hanns Eisler. Gesammelte Schriften 19211935, hg. von Tobias Faßhauer und Günter Mayer, Wiesbaden: Breitkopf \& Härtel, 2007, S. $155 \mathrm{f}$.

Eisler, Hanns: Dumpfe Trommel und berauschtes Gong [1917], in: Lieder für Singstimme und Klavier 1917-1921, hg. von Julia Rittig-Becker und Christian M. Schmidt, Wiesbaden: Breitkopf \& Härtel, 2009 (Gesamtausgabe. Serie 3, Bd. 1), S. $2-8$.

Eisler, Hanns: [Wiener Tagebuch 1921-1922], in: Hanns Eisler. Gesammelte Schriften 19211935, hg. von Tobias Faßhauer und Günter Mayer, Wiesbaden: Breitkopf \& Härtel, 2007, S. $1-22$.

Hanheide, Stefan: Wahrnehmungen von Kriegstragik im Kunstlied während des Ersten Weltkriegs, in: Musik bezieht Stellung. Funktionalisierungen der Musik im Ersten Weltkrieg, hg. von Stefan Hanheide, Dietrich Helms, Claudia Glunz und Thomas F. Schneider, Göttingen: Universitätsverlag Osnabrück, 2013, S. 307-332.

Hindrichs, Gunnar: Philosophie der Revolution, Berlin: Suhrkamp, 2017.

http://klabund.eu/ (Zugriff am 06.08.2020).

Kraus, Karl: Kriegsmüde, in: Die Fackel 20 (1918), Nr. 474-483, S. 153.

Lethen, Helmut: Verhaltenslehren der Kälte. Lebensversuche zwischen den Kriegen, Frankfurt am Main: Suhrkamp, 2014.

Jian, Ming: Expressionistische Nachdichtungen chinesischer Lyrik, Frankfurt am Main: Peter Lang, 1990.

Jünger, Ernst: In Stahlgewittern, Stuttgart: Klett-Cotta, 2013.

Lenin, Wladimir Iljitsch: Staat und Revolution. Die Lehre des Marxismus vom Staat und die Aufgaben des Proletariats in der Revolution, in: Lenin. Werke, Bd. 25, Berlin: Dietz, 1968, S. 393-507. 
Narveson, Jan: Pacifism. A philosophical Analysis, in: Ethics 75 (1965), S. 259-271, https://doi.org/10.1086/291549.

Pan-Hsu, Kuei-Fen: Die Bedeutung der chinesischen Literatur in den Werken Klabunds. Eine Untersuchung zur Entstehung der Nachdichtungen und deren Stellung im Gesamtwerk, Frankfurt am Main: Peter Lang, 1990.

Phleps, Thomas: »Der müde Soldat«. Hanns Eisler und der Erste Weltkrieg, in: Musik bezieht Stellung. Funktionalisierungen der Musik im Ersten Weltkrieg, hg. von Stefan Hanheide, Dietrich Helms, Claudia Glunz und Thomas F. Schneider, Göttingen: Universitätsverlag Osnabrück, 2013, S. 403-428.

Schmidt, Christian Martin: Einleitung, in: Hanns Eisler: Lieder für Singstimme und Klavier 1917-1921, hg. von Julia Rittig-Becker und Christian M. Schmidt, Wiesbaden: Breitkopf \& Härtel, 2009 (Gesamtausgabe. Serie 3, Bd. 1), S. XI-XVI.

Christoph Haffter studierte Philosophie und Musikwissenschaft in Basel, Paris, Berlin und New York. Er promoviert an der Universität Basel mit einer Arbeit zur Ästhetik der Gegenwartsmusik und ist Assistent am Lehrstuhl für Kunstphilosophie der Universität Fribourg. 


\title{
Der doppelte Po und die Musik
}

Rätoromanisch-chinesische Studien, besonders zu

Li Po, Harry Partch und Chasper Po

\author{
Herausgegeben von \\ Mathias Gredig, Marc Winter, \\ Rico Valär und Roman Brotbeck \\ Redaktionelle Mitarbeit \\ Daniel Allenbach
}

Königshausen \& Neumann 
Bibliografische Information der Deutschen Nationalbibliothek

Die Deutsche Nationalbibliothek verzeichnet diese Publikation in der Deutschen

Nationalbibliografie; detaillierte bibliografische Daten sind im Internet über http://dnb.d-nb.de abrufbar.

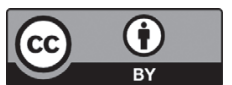

Dieses Werk ist lizenziert unter der Creative Commons Attribution 4.0 Lizenz (BY). Diese Lizenz erlaubt unter Voraussetzung der Namensnennung des Urhebers die Bearbeitung, Vervielfältigung und Verbreitung des Materials in jedem Format oder Medium für beliebige Zwecke, auch kommerziell. (Lizenztext: https://creativecommons.org/ licenses/by/4.0/deed.de) Die Bedingungen der Creative-Commons-Lizenz gelten nur für Originalmaterial. Die Wiederverwendung von Material aus anderen Quellen (gekennzeichnet mit Quellenangabe) wie z. B. Schaubilder, Abbildungen, Fotos und Textauszüge erfordert ggf. weitere Nutzungsgenehmigungen durch den jeweiligen Rechteinhaber.

Erschienen 2021 im Verlag Königshausen \& Neumann GmbH

(C) bei den Autoren

Die Druckvorstufe dieser Publikation wurde vom Schweizerischen Nationalfonds zur Förderung der wissenschaftlichen Forschung unterstützt.

\section{FNSNF}

SCHWEIZERISCHER NATIONALFONDS

ZUR FÖRDERUNG DER WISSENSCHAFTLICHEN FORSCHUNG

Wir danken der Kulturförderung des Kantons Graubünden.

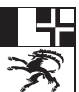

Kulturförderung Graubünden. Amt für Kultur

Promoziun da la cultura dal Grischun. Uffizi da cultura

Promozione della cultura dei Grigioni. Ufficio della cultura

SWIISSLOS

Hochschule der Künste Bern

www.hkb.bfh.ch

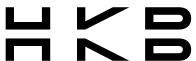

Hochschule der Künste Bern

Haute école des arts de Bern

Bern University of the Arts

Umschlag: skh-softics / coverart

Umschlagabbildung: Lea Gredig

Print-ISBN 978-3-8260-7180-5

PDF-ISBN 978-3-8260-7233-8

DOI $10.26045 /$ po

https.//doi.org/10.36202/9783826072338

Gedruckt auf säurefreiem, alterungsbeständigem Papier

Printed in Germany

www.koenigshausen-neumann.de

www.ebook.de

www.buchhandel.de

www.buchkatalog.de 


\section{Inhalt}

Prolog

Dumenic Andry

Chasper Pos Humor

Renzo Caduff

Chasper Pos rhythmische Versgestaltung - eine shinkende Mähre`?

Rico Valär

Rätoromanische Nachdichtungen chinesischer Lyrik bei

Gian Fadri Caderas und Peider Lansel

Eine Spurensuche

Mathias Gredig

China in rätoromanischen Zeitungen, Zeitschriften

und literarischen Texten

Marion Eggert

Schwalbenflug in Gedichten von Li Bai und Chasper Po

Thomas Geissmann

Die Rolle der Gibbons beim chinesischen Dichter Li Bai

Marc Winter

„Chinas Dichterfürst«

Die Rezeption Li Bais als literarischer Superstar im Westen

Eva Schestag

"A most difficult man«

Ezra Pound als Übersetzer von Li Bai, mit einem Seitenblick auf Shigeyoshi Obata

Odila Schröder

Chinesische Li-Bai-Vertonungen in Jahren der Unruhe 
Mathias Gredig

Quantitative Überlegungen zum Phänomen

der Li-Bai-Vertonungen im Westen

Mit Beobachtungen zu drei Vertonungen des Gedichtes

Chun ye Luo cheng wen di (In einer Frühlingsnacht in Luoyang eine Flöte hören)

\section{Gesine Schröder}

"Die Hüften schwingen sich nun nicht mehr»

Li-Bai-Vertonungen von Komponistinnen

Heinrich Aerni

Li-Bai-Vertonungen in der Schweiz

Matthias Schmidt

Übersetzung ohne Original?

Gustav Mahler, Anton Webern und Li Bai

\section{Christoph Haffter}

Szenen der Selbstenttäuschung

Hanns Eislers Die rote und die weiße Rose nach Li Bai und die Antinomien der Kriegslyrik

Thomas Meyer

„Wunderlich im Spiegelbilde»

Zu einigen Vertonungen des Pavillon-Gedichts

\section{Mathias Gredig}

Gedanken über Li Bais Jing ye si (Gedanken in einer stillen Nacht)

und dessen Vertonungen im Westen

\section{Martin Skamletz}

"I've turned into a great reviser."

Lee Hoibys Vertonung von Li Bais The River-Merchant's Wife: A Letter

und ihr Bezug zu Harry Partch

\section{Martin Skamletz}

"Of course I am a weak shadow of Lee Hoiby as a Kitharist."

Five letters by Harry Partch, 1948-1958

\section{Marc Kilchenmann}

Ben Johnstons Verhältnis zu Harry Partch und seine Three Chinese Lyrics 
Eleni Ralli

Parallelen und Modifikationen der Notation in verschiedenen Quellen

von Harry Partchs Seventeen Lyrics by Li Po

Schwierigkeiten und Transkriptionsvorschläge

Charles Corey

Gesture and Intention in the Art Songs of Harry Partch

Caspar Johannes Walter

Sprechmelodie als Quelle von Melodik und Harmonik

The Intruder aus Harry Partchs Li-Bai-Vertonungen

Roman Brotbeck

Der Sprechgesang bei Arnold Schönberg und Harry Partch

Eine Annäherung

Namensregister 